\title{
Erratum to: An R package to compute commonality coefficients in the multiple regression case: An introduction to the package and a practical example
}

\author{
Kim Nimon $^{1}$ • Mitzi Lewis ${ }^{2} \cdot$ Richard Kane $^{3} \cdot$ R. Michael Haynes ${ }^{1}$
}

Published online: 31 January 2017

(C) Psychonomic Society, Inc. 2017

Erratum to: Behav Res 2008, 40 (2), 457-466

DOI:10.3758/BRM.40.2.457

On page 458 of the article, the formula for $\mathrm{C}(\mathrm{ij}), \mathrm{C}(\mathrm{ik})$, and $\mathrm{C}(\mathrm{jk})$ in Table 1 contain an error. On the same page, the formula for $\mathrm{C}(\mathrm{ij})$ in Table 2 contains an error. The corrected tables are shown below.

Table 1 Unique and Commonality Formulas for Three Predictor Variables

Three Predictor Variables

$\mathrm{U}_{(\mathrm{i})}=\mathrm{R}_{\mathrm{y} . \mathrm{ijk} \mathrm{k}}^{2}-\mathrm{R}_{\mathrm{y} . \mathrm{jk}}^{2}$

$\mathrm{U}_{(\mathrm{j})}=\mathrm{R}_{\mathrm{y} \cdot \mathrm{ijk}}^{2}-\mathrm{R}_{\mathrm{y} \cdot \mathrm{ik}}^{2}$

$\mathrm{U}_{(\mathrm{k})}=\mathrm{R}_{\mathrm{y} . \mathrm{jik}}^{2}-\mathrm{R}_{\mathrm{y} . \mathrm{ij}}^{2}$

$C_{(i j)}=R_{y . i k}^{2}+R_{y . j k}^{2}-R_{y . k}^{2}-R_{y . j i k}^{2}$

$C_{(i k)}=R_{y . j j}^{2}+R_{y . j k}^{2}-R_{y . j}^{2}-R_{y . j i k}^{2}$

$C_{(j k)}=R_{y . j i}^{2}+R_{y . i k}^{2}-R_{y . i}^{2}-R_{y . j i k}^{2}$

$C_{(i j k)}=R_{y . i}^{2}+R_{y . j}^{2}+R_{y . k}^{2}-R_{y . j i j}^{2}-R_{y . i k}^{2}-R_{y . j k}^{2}+R_{y . j i k}^{2}$

The online version of the original article can be found at http://dx.doi. org/10.3758/BRM.40.2.457.

Kim Nimon

knimon@uttyler.edu

Southern Methodist University, Dallas, Texas, USA

2 University of North Texas, Denton, Texas, USA

3 University of North Florida, Jacksonville, Florida, USA
Table 2 Unique and Commonality Formulas for Four Predictor Variables

Four Predictor Variables

$\mathrm{U}_{(\mathrm{i})}=\mathrm{R}_{\mathrm{y} . \mathrm{ijk} \mathrm{l}}^{2}-\mathrm{R}_{\mathrm{y} . \mathrm{jk} 1}^{2}$

$U_{(j)}=R_{y . j i k l}^{2}-R_{y . i k l}^{2}$

$\mathrm{U}_{(\mathrm{k})}=\mathrm{R}_{\mathrm{y} . \mathrm{ijkl}}^{2}-\mathrm{R}_{\mathrm{y} . \mathrm{jil} 1}^{2}$

$\mathrm{U}_{(\mathrm{l})}=\mathrm{R}_{\mathrm{y} . \mathrm{j} \mathrm{jk}}^{2}-\mathrm{R}_{\mathrm{y} \cdot \mathrm{ijk}}^{2}$

$C_{(i j)}=-R_{y . k l}^{2}+R_{y . j k l}^{2}+R_{y . j k l}^{2}-R_{y . j i k l}^{2}$

$C_{(i k)}=-R_{y . j l}^{2}+R_{y . j i j l}^{2}+R_{y . j k l}^{2}-R_{y . j i j k 1}^{2}$

$C_{(i l)}=-R_{y . j k}^{2}+R_{y . j i k}^{2}+R_{y . j k l}^{2}-R_{y . j i k l}^{2}$

$C_{(j \mathrm{k})}=-R_{y . i l}^{2}+R_{y . j i j l}^{2}+R_{y . i k l}^{2}-R_{y . j i j k}^{2}$

$C_{(\mathrm{jl})}=-R_{y . i k}^{2}+R_{y . j i k k}^{2}+R_{y . i k l}^{2}-R_{y . j i k l}^{2}$

$\mathrm{C}_{(\mathrm{kl})}=-\mathrm{R}_{\mathrm{y} . \mathrm{ij}}^{2}+\mathrm{R}_{\mathrm{y} . \mathrm{ijk}}^{2}+\mathrm{R}_{\mathrm{y} . \mathrm{ijl}}^{2}-\mathrm{R}_{\mathrm{y} . \mathrm{ijk} \mathrm{l}}^{2}$

$C_{(i j k)}=-R_{y .1}^{2}+R_{y . i l}^{2}+R_{y . j l}^{2}+R_{y . k l}^{2}-R_{y . j i l}^{2}-R_{y . j k l}^{2}-R_{y . j k l}^{2}+R_{y . j i k l}^{2}$

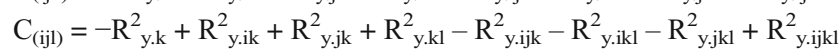

$C_{(i k l)}=-R_{y . j}^{2}+R_{y . j j}^{2}+R_{y . j k}^{2}+R_{y . j l}^{2}-R_{y . j k k}^{2}-R_{y . j i j l}^{2}-R_{y . j k l}^{2}+R_{y . j i k l}^{2}$

$C_{(j k l)}=-R_{y . i}^{2}+R_{y . j j}^{2}+R_{y . i k}^{2}+R_{y \cdot i l}^{2}-R_{y . j i k}^{2}-R_{y . j i l}^{2}-R_{y . i k l}^{2}+R_{y \cdot j k l}^{2}$

$C_{(i j k l)}=R_{y . i}^{2}+R_{y . j}^{2}+R_{y . k}^{2}+R_{y .1}^{2}-R_{y \cdot i j}^{2}-R_{y, i k}^{2}-R_{y \cdot i l}^{2}-R_{y . j k}^{2}-R_{y . j 1}^{2}-$

$R_{y . k l}^{2}+R_{y . j i k}^{2}+R_{y . j i l}^{2}+R_{y . i k l}^{2}+R_{y . j k l}^{2}-R_{y . j i k l}^{2}$ 